Apidologie, 1978, $9(1), 1-17$.

\title{
ORIGINE GÉOGRAPHIQUE DE LA GELÉE ROYALE
}

\section{Die geographische Herkunft des Weiselfuttersaftes}

\author{
Giancarlo RICCIARDELLI d'ALBORE \\ et \\ Marcella BATTAGLINI BERNARDINI \\ Istituto di Zoocolture della Università_di Perugia (Italie)
}

SUMMARY

GEOGRAPHICAL ORIGIN OF ROYAL JELLY

A palynological research was carried out in order to verify the possibility of establishing the geographical origin of royal jelly by means of the pollen spectrum study.

The analysis of 106 samples from 12 countries pointed out that this possibility exists and the method can be easily used for checking the yields commercialized on the international scale.

\section{RÉSUMÉ}

Une recherche palynologique a été effectuée dans le but de vérifier s'il était possible de définir l'origine géographique de la gelée royale grâce à l'étude de son spectre pollinique.

Les analyses de 106 échantillons provenant de 12 pays ont prouvé que cette méthode était valable et facilement utilisable pour contrôler les productions commerciales à l'échelle internationale.

\section{INTRODUCTION}

Tout le monde est d'accord pour considérer la mélissopalynologie comme un moyen efficace pour remonter à l'origine géographique d'un miel, mais peu de gens savent que la gelée royale aussi présente un spectre pollinique variable par rapport à sa zone de production. Ce furent Louveaux et Chauvin en 1956 qui mirent en évidence la possibilité de remonter au lieu de provenance de la gelée royale à travers 
l'étude des pollens présents dans la gelée royale même. Depuis le sujet n'a pas été reconsidéré, peut-être parce que le commerce de ce produit n'intéresse encore qu'un nombre limité de pays.

Si la production, et donc les échanges, subissent une augmentation, comme cela est prévisible, une réglementation adéquate sera nécessaire, vu que déjà actuellement on trouve des gelées royales à des degrés de pureté divers et à des prix extrêmement variables, qui souvent ne reflètent pas les caractéristiques qualitatives du produit.

C'est dans cet esprit qu'on a conduit cette recherche, avec la conviction que les finalités d'ordre purement scientifique pourront sous peu trouver résonance dans un domaine pratique.

\section{MATÉRIEL ET MÉTHODES}

Grâce à l'aimable collaboration de spécialistes et d'apiculteurs de nombreux pays, on a analysé 106 échantillons de gelée royale qui provenaient de : Chine (2), Mexique (3), Texas (1), Canada (2), Russie (2), Pologne (2), France (3), Roumanie (4), Yougoslavie (1), Tchécoslovaquie (2), Bulgarie (4) et Italie (80).

Pour les analyses on a adopté la méthode indiquée ci-dessous : $0,5 \mathrm{~g}$ de gelée royale, dissous dans $8 \mathrm{ml}$ d'eau distillée, sont homogénéisés pendant $1 \mathrm{mn}$ par un agitateur électromagnétique. On rend limpide la solution en neutralisant, goutte à goutte, avec $\mathrm{KOH}$ à $5 \%$ et on agite de nouveau pendant $30 \mathrm{~s}$; puis on centrifuge dans une éprouvette de $10 \mathrm{ml}$, pendant $10 \mathrm{mn}$ à $3000 \mathrm{trs} / \mathrm{mn}$. Après décantation on ajoute $10 \mathrm{ml}$ d'une solution à $0,1 \mathrm{~N}_{\text {de }} \mathrm{H}_{2} \mathrm{SO}_{4}$, on centrifuge de nouveau, on décante et on transfère enfin le sédiment sur une lame. Pour l'équipement de la préparation on procède comme pour les miels.

\section{DISCUSSION DES RÉSULTATS}

Il faut tout d'abord remarquer que l'analyse pollinique de la gelée royale est bien plus difficile que celle du miel, puisque dans le sédiment on retrouve un pourcentage plus ou moins élevé de grains de pollen en fragments ou altérés (fossilisés ou partiellement digérés). Dans le cas des fragments il est possible quelquefois de remonter au genre botanique si leurs dimensions ne sont pas trop exiguës; pour les grains altérés, au contraire, la classification est souvent problématique puisqu'il s'agit pour la plus grande part de pollens digérés.

Pour les pollens fossilisés enfin, il suffit de connaitre la palynologie fossile (ERDTMAN et al.) pour en déterminer l'espèce.

Les résultats sont de toute façon suffisamment fiables même si l'on se rapporte au domaine des pollens normaux, provenant de pollution (poils et pattes des nourrices), qui représentent un remarquable pourcentage.

L'examen microscopique des 106 échantillons de gelée royale a mis en évidence les spectres suivants :

ITALIE. Les productions viennent essentiellement de la plaine du Pô, des collines 
environnantes et des Préalpes; on peut également obtenir de petites quantités dans d'autres zones, telles que l'île d'Elbe et l'Italie du centre.

La gelée royale de l'île d'Elbe (Fig. 1) est caractérisée par un spectre pollinique typique du milieu méditerranéen : Lavandula Stoechas L., Rubus fruticosus L., Clematis Vitalba L., Eucalyptus sp., Myrtus communis L., Castanea sativa Mill., Cistus incanus L. et Cistus monspeliensis L., Erica sp.

Les échantillons provenant de la plaine du Pô ont été récoltés pour une bonne part pendant toute la période de production (juin-septembre).

De l'ensemble des analyses il en est ressorti sept spectres polliniques principaux :

1) Lotus sp., Vitis vinifera L. (\% élevé), Trifolium repens L., Rumex sp., Daucus carota L., Zea Mays L., Amaranthus sp., Quercus Robur L.;

2) Onobrychis viciaefolia Scop. (\% élevé), Trifolium repens, Vitis, Papaver Rhoeas L., Plantago sp., Crataegus Oxyacantha L., Coronilla sp., Gleditschia triacanthos L.;

3) Castanea, Helianthus sp. (\% élevé), Trifolium repens, Trifolium pratense L., Vitis, Zea, Plantago.

4) Brassica sp. (\% élevé), Trifolium repens, Clematis, Vitis (\% élevé), Rumex, Lotus, Vicia sp.;

5) Tordylium apulum L. (\% élevé), Hedysarum coronarium L., Fraxinus sp., Carex sp., Vitis;

6) Papaver, Rubus, Mercurialis sp. (\% élevé), Trifolium repens, Amaranthus, Hedysarum;

7) Castanea (\% élevé), Paliurus australis Gaertn., Vitis, Cornus sanguinea L., Artemisia sp. (\% élevé), Coronilla, Daucus, Hedysarum.

Il faut remarquer la présence du pollen de Vitis (qu'on n'a jamais relevée dans les échantillons des autres pays) qui peut aussi atteindre des pourcentages considerrables; on peut en déduire que dans la plaine du Pô les abeilles recueillent du pollen sur les vignes.

CHINE (Fig. 3). Le spectre pollinique de la gelée royale chinoise est particulier et rappelle celui du miel. Il contient peu d'espèces : Astragalus sinicus, Vicia, Brassica, Ombellifères. Le pollen d'Astragalus peut représenter $90 \%$.

MEXIQUE. On a déterminé 4 spectres polliniques, eux aussi très caractéristiques :

1) Mimosa bimucronata, Cruciferae, Citrus sp., Echium sp., Myrtaceae, Cactaceae, Croton sp.;

2) Bursera sp., Bombax sp., Prosopis sp.;

3) Tiliaceae, Caesalpiniaceae, Bravaisia sp., Mimosaceae, Bursera, Rhus sp. (Fig. 4);

4) Myrtaceae, Galega Gr., Bursera, Vigueria, Prosopis. 


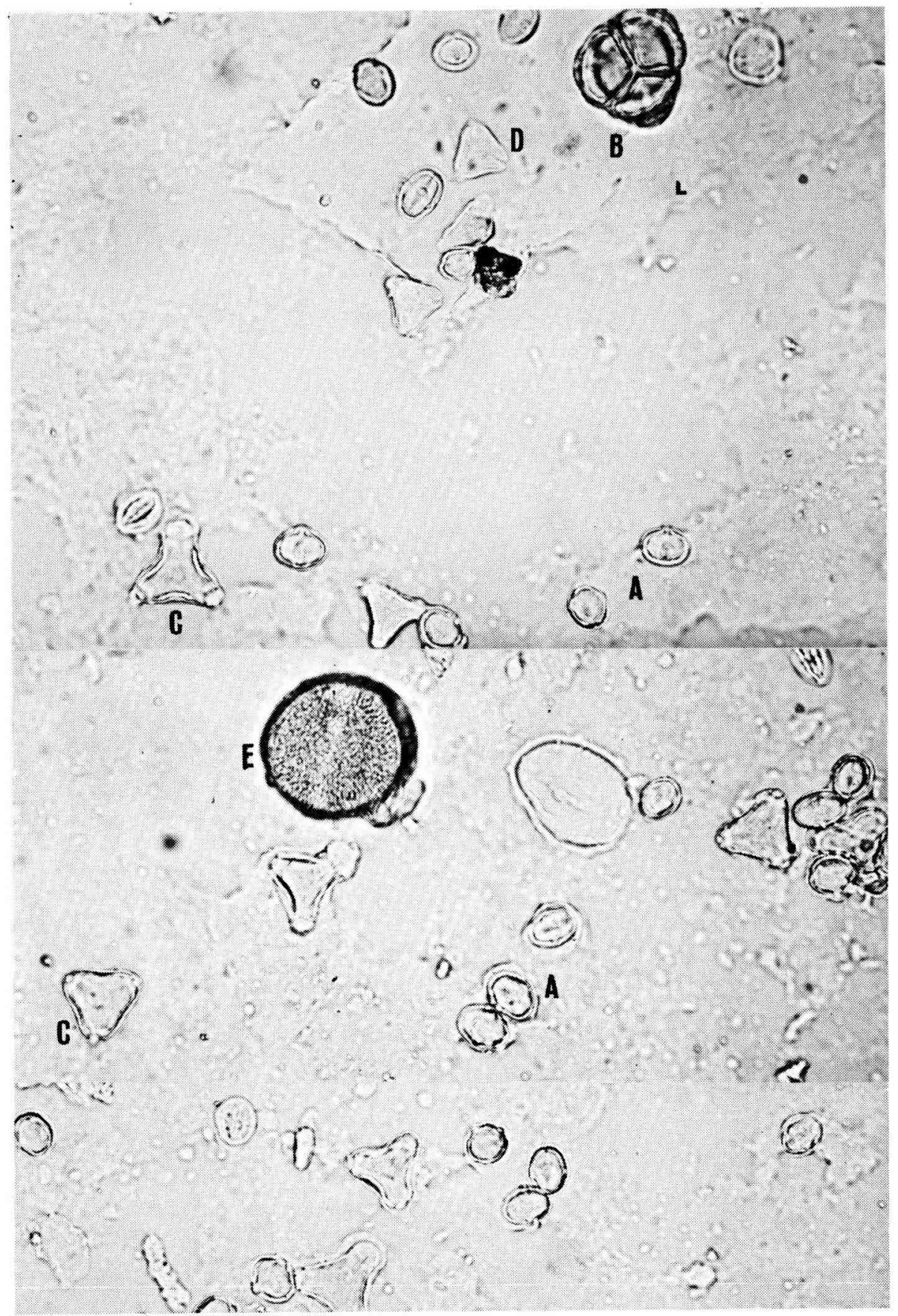

FIG. 1. - Spectre pollinique d'une gelée royale d'Italie.

A) Castanea sativa Mill.; B) Erica arborea $L . ;$ C) Eucalyptus sp.; D) Myrtus communis L.; E) Cistus monspeliensis $L$.

Aвв. 1. - Pollenspektrum eines Weiselfuttersaftes aus Italien. 


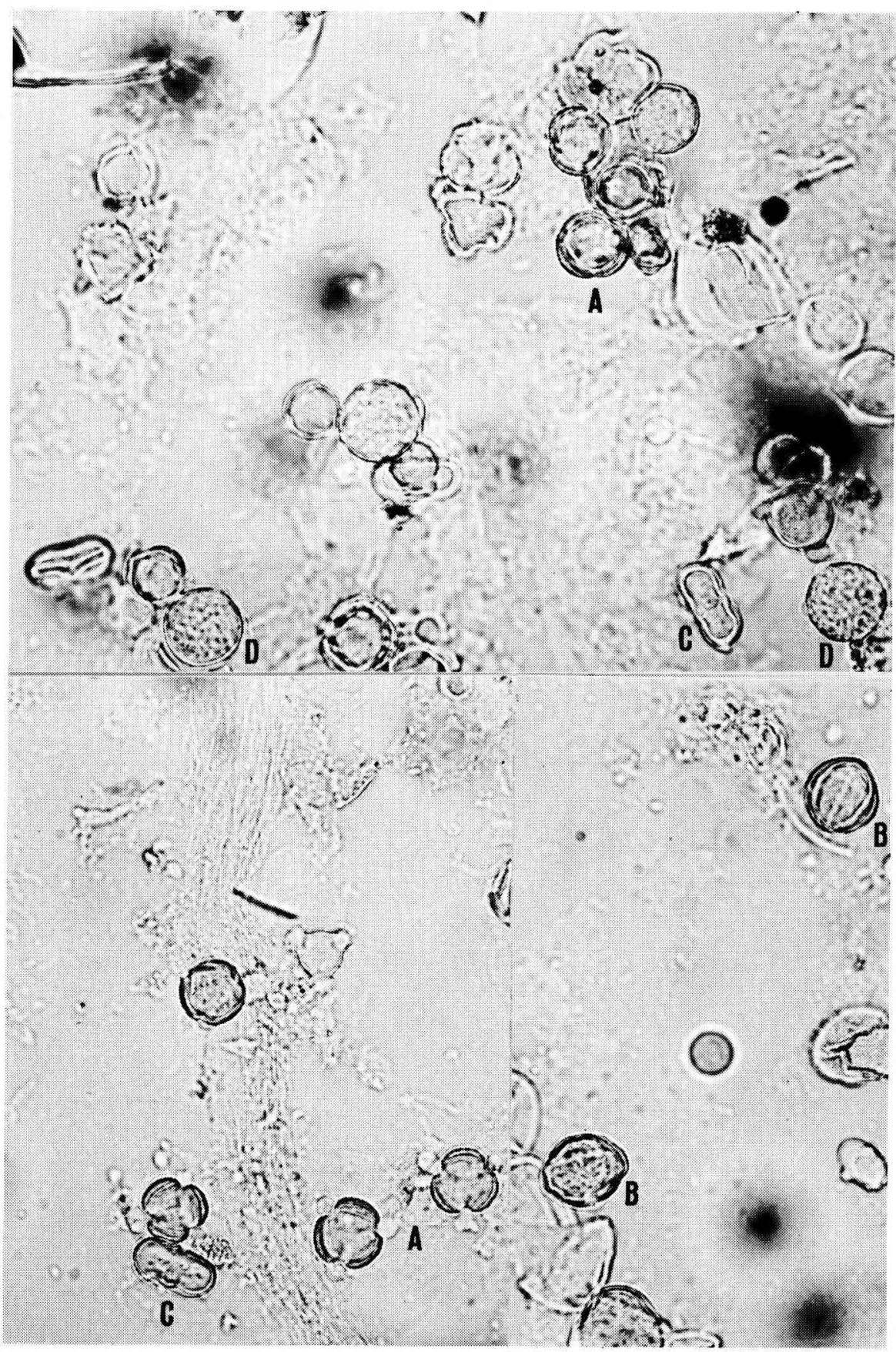

FIG. 2. - Spectre pollinique d'une gelée royale d'Italie.

A) Artemisia vulgaris $L . ; B$ ) Vitis vinifera $L . ; C)$ Pimpinella $s p . ; D$ ) Trifolium hibridum $L$.

Авв. 2. - Pollenspektrum eines Weiselfuttersaftes aus Italien. 


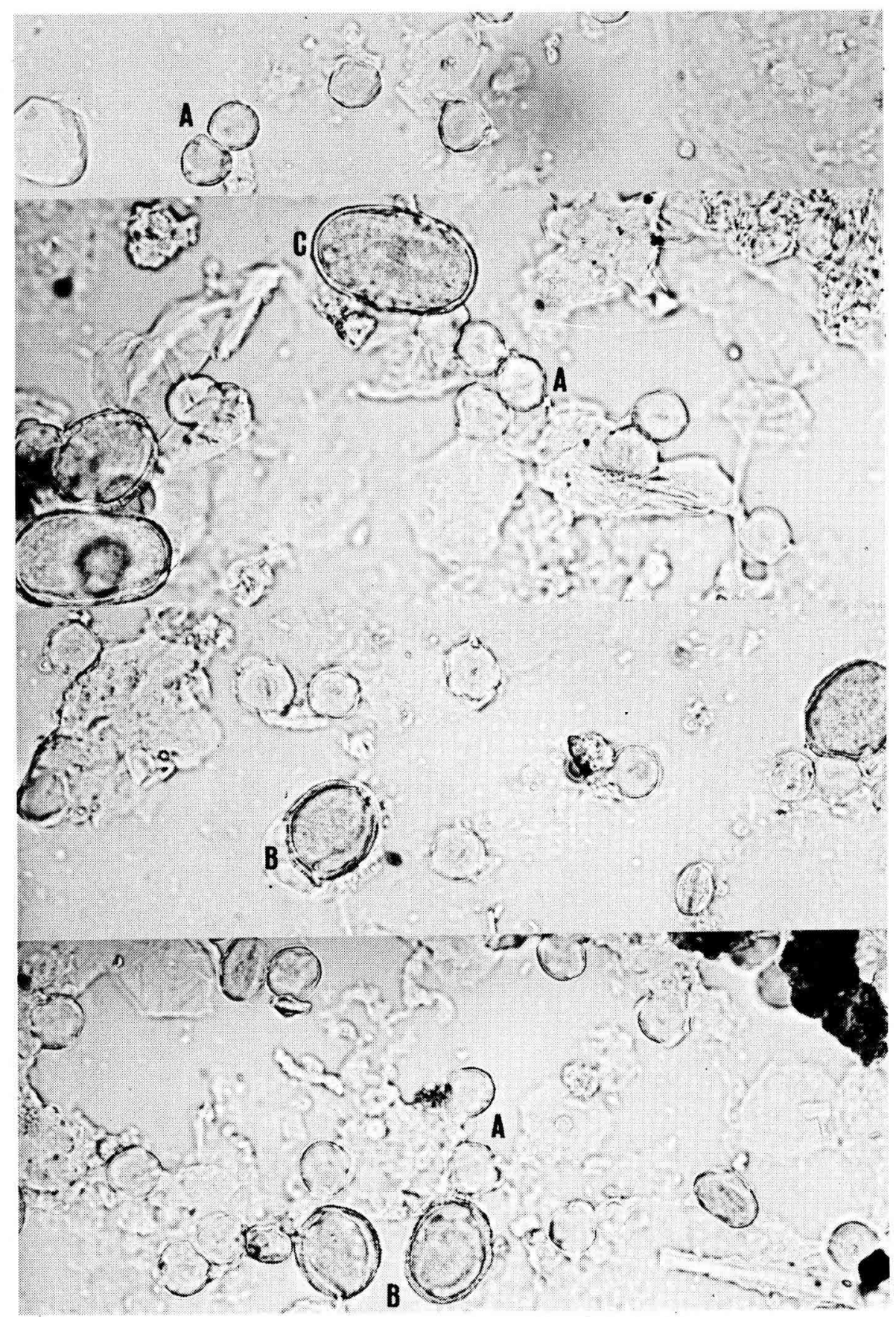

FIG. 3. - Spectre pollinique d'une gelée royale de Chine.

A) Astragalus sinicus; $B$ ) Brassica $s p$. C) Vicia Faba $L$.

Aвв. 3. - Pollenspektrum eines Weiselfuttersaftes aus China. 


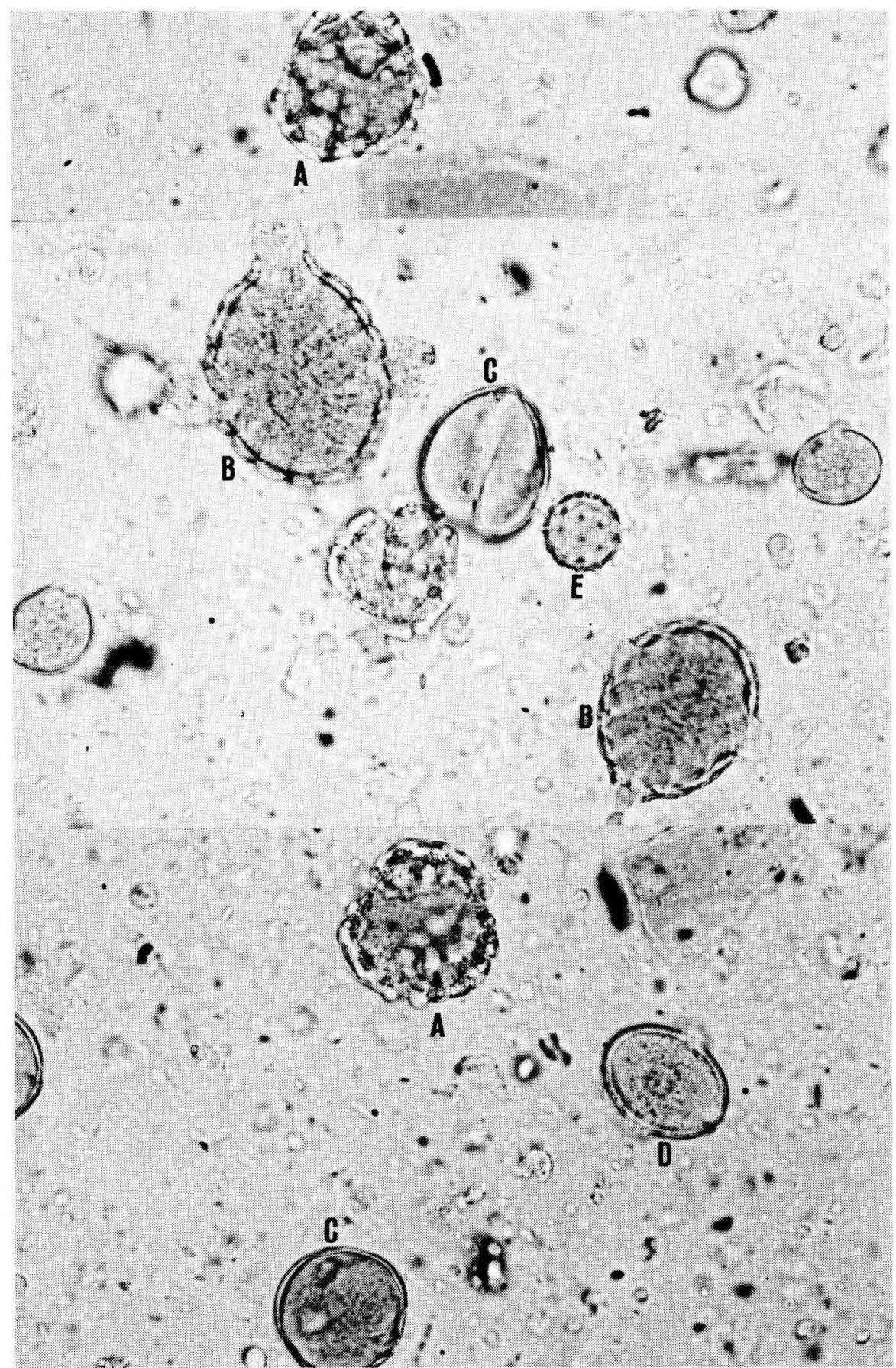

Fio. 4. - Spectre pollinique d'une gelée pợale du Mexique.

A) Caesalpinia sp.; B) Bravaisia Gr.; C) Julbernardia Gr.; D) Tiliacée; E) Bidens Gr.

Aв8. 4. - Pollenspektrum eines Weiselfuttersaftes aus Mexiko. 
Les pollens sont typiques des milieux tropicaux ou subtropicaux et on les trouve aussi dans les miels de ces zones.

TEXAS (U.S.A.) (Fig. 5). Il présente la combinaison Mimosa, Graminées, Echinocactus sp., Caesalpinia sp., Palmae, Epilobium Gr., Eucalyptus et d'autres Myrtaceae, Solanaceae, Vernonia sp. et un petit pollen morphologiquement semblable au pollen de Smilax aspera $\mathrm{L}$.

Dans les autres gelées royales on a relevé les spectres polliniques indiqués à côté de chaque pays.

ONTARIO (Canada) (Fig. 6). Lotus (\% élevé, 2 formes), Pirus sp., Cruciferae, Melilotus sp. (\% élevé), Trifolium repens, Centaurea sp., Labiatae M., Magnolia sp.

RUSSIE (Fig. 7). Phacelia tanacetifolia, Melilotus, Cruciferae, Caryophyllaceae, Centaurea Cyanus L., Convolvulus sp.; il faut remarquer le pollen de Phacelia présent en $\%$ très élevé (65\%).

RUSSIE (Caucase) (Fig. 8). Polygonum Fagopyrum L. (\% élevé), Rubus, Umbelliferae, Centaurea Cyanus, Trifolium pratense, Helianthus annuus L., Carduus sp., Verbascum sp., Tilia sp., Arctium sp., Stachys sp., Sambucus nigra L.

POLOGNE. Campanula sp., Vicia sp. (\% élevé), Cruciferae (\% élevé), Tilia, Prunus sp., Centaurea Cyanus, Galega, Lychnis sp., Rubus, Cornus.

ROUMANIE (Fig. 9). Helianthus (\% élevé), Heracleum sp., Centaurea Cyanus, Carduus, Taraxacum officinale Weber, Xanthium sp., Hedera Helix L., Tilia, Aesculus sp., Trifolium repens, Phacelia.

YOUGOSLAVIE (Fig. 10). Tilia, Dipsacacea, Compositae H (Senecio sp. et Aster sp. en \% élevé), Olea europea L., Castanea, Galium sp., Rhamnus, Campanula, Pimpinella sp., Thymus sp., Ononis sp.

TCHÉCOSLOVAQUIE (Fig. 11). Acer sp. (\% élevé), Tilia, Centaurea Cyanus, Lonicera sp., Taraxacum, Pirus, Galega, Cruciferae, Ranunculus sp., Trifolium incarnatum L., Melilotus, Salix.

BULGARIE. Umbelliferae, Tilia, Cruciferae, Trifolium repens, Salix, Prunus, Helianthus, Cucurbita Pepo L., Epilobium, Castanea, Brassica, Convolvulus, Echium, Polygonum.

FRANCE. Brassica, Castanea, Umbelliferae, Onobrychis, Rubus, Pirus, Compositae A et H, Trifolium pratense, Centaurea Jacea L., Ambrosia sp., Papaver, Rumex.

\section{CONCLUSIONS}

Les spectres polliniques énumérés montrent de façon significative qu'il est possible de différencier les gelées royales selon leur pays d'origine. 


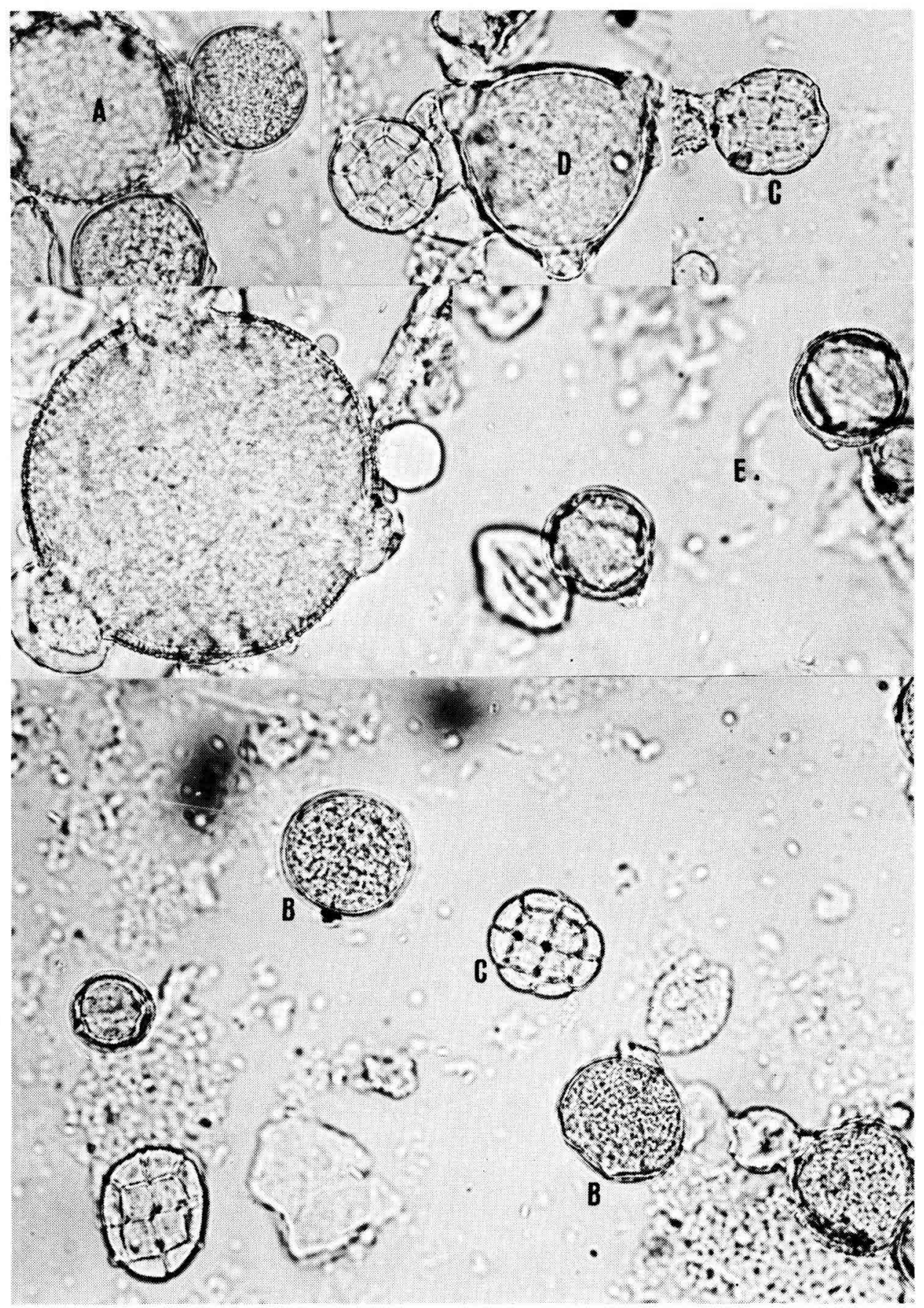

Fig. 5. - Spectre pollinique d'une gelée royale du Texas.

A) Caesalpinia Gr.; B) Graminacée; C) Mimosa $g r . ; D)$ Epilobium $s p . ; E)$ Solanacée. Aв8. 5. - Pollenspektrum eines Weiselfuttersaftes aus Texas. 


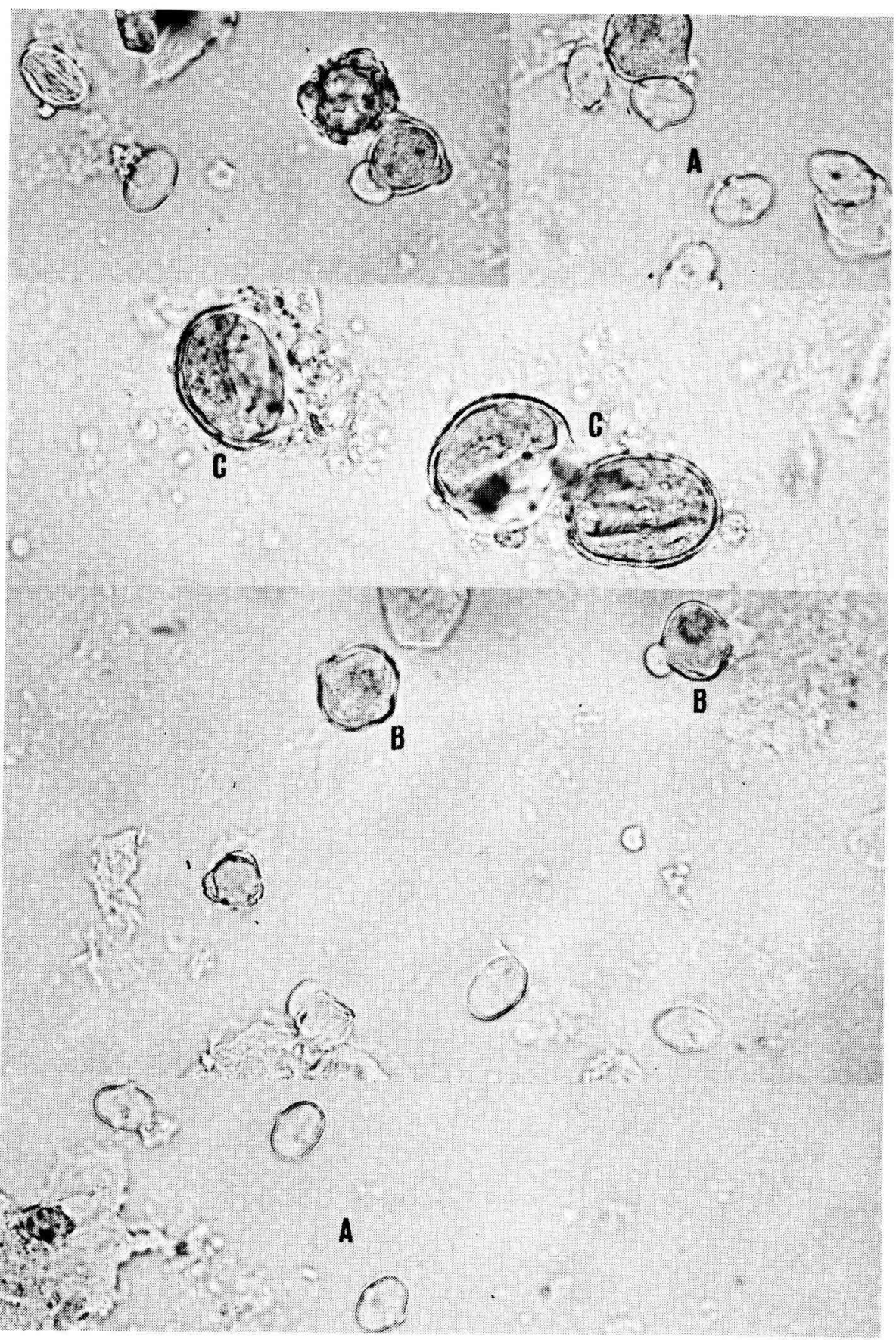

Fig. 6. - Spectre pollinique d'une gelée royale du Canada.

A) Lotus $s p . ; B)$ Trifolium repens $L . ; C)$ Magnolia $s p$.

Aв8. 6. - Pollenspektrum eines Weiselfuttersaftes aus Kanada. 


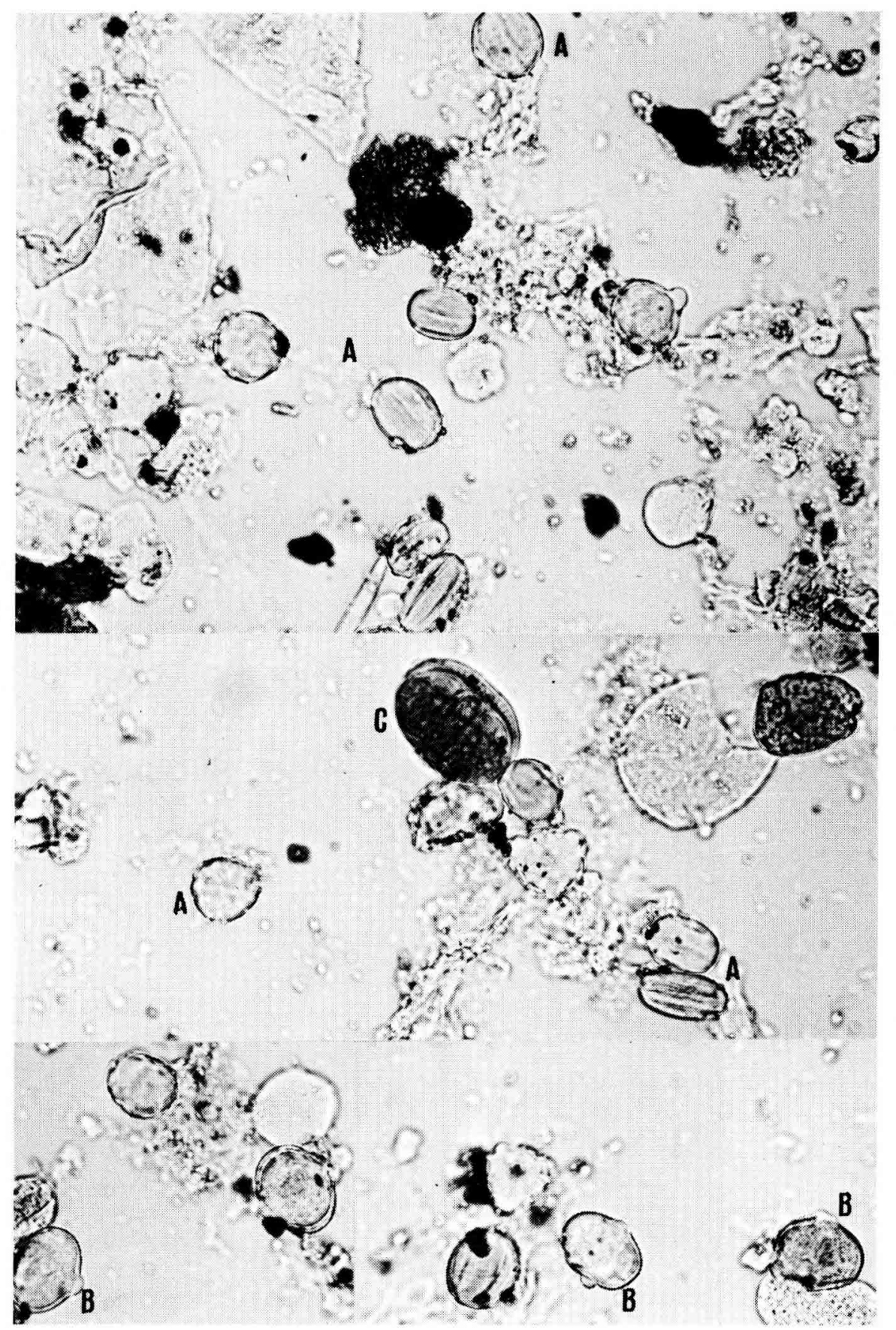

FIG. 7. - Spectre pollinique d'une gelée royale de Russie.

A) Phacelia tanacetifolia; $B$ ) Melilotus $s p . ;$ C) Centaurea Cyanus $L$.

Aвв. 7. - Pollenspektrum eines Weiselfuttersaftes aus Russland. 


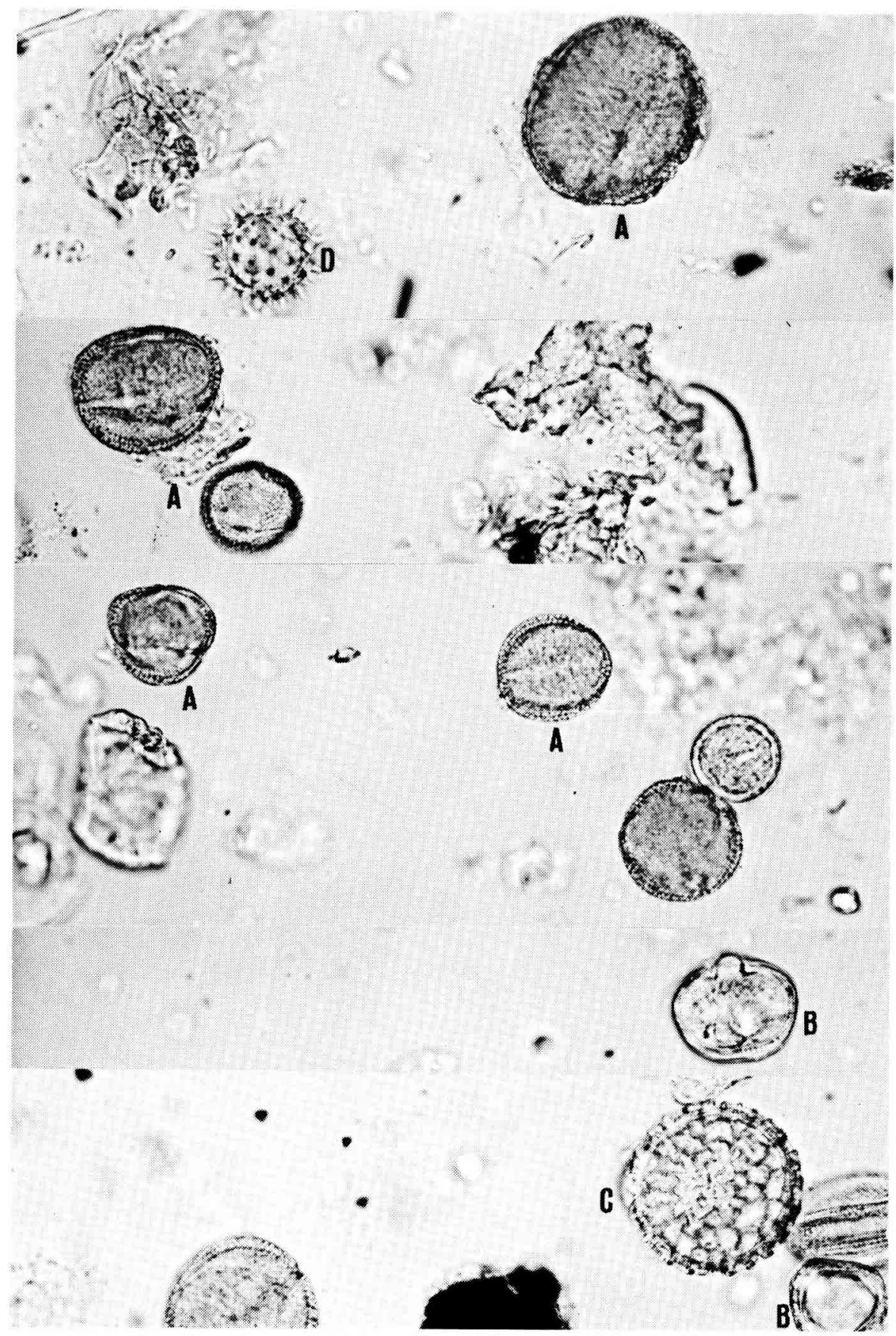

Fig. $\hat{8}$. - Sipectre poilinique äune geiée royaile de Russie (Caucase). A) Polygonum Fagopyrum L.; B) Centaurea Cyanus $L . ; C$ ) Polygonum $s p$. $D$ ) Helianthus annuus $L$.

Aвв. 8. - Pollenspektrum eines Weiselfuttersaftes aus Russland (Kaukasus). 


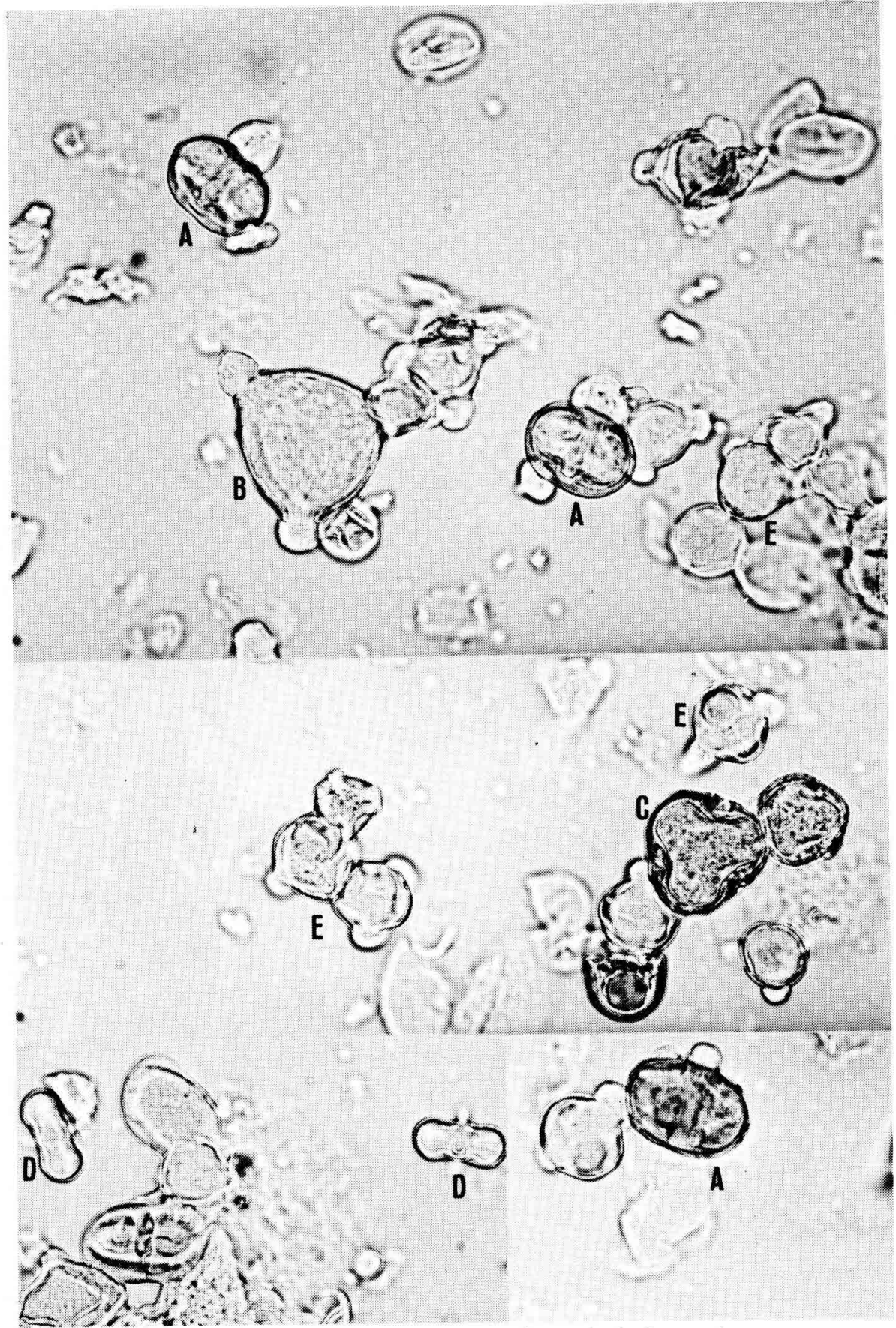

FIG. 9. - Spectre pollinique d'une gelèe royale de Roumanie.

A) Centaurea Cyanus $L . ; B$ ) Lonicera $s p$.; C) Tilia $s p . ; D)$ Ombellifère; $E$ ) Trifolium repens $L$.

Авв. 9. - Pollenspektrum eines Weiselfuttersaftes aus Rumänien. 


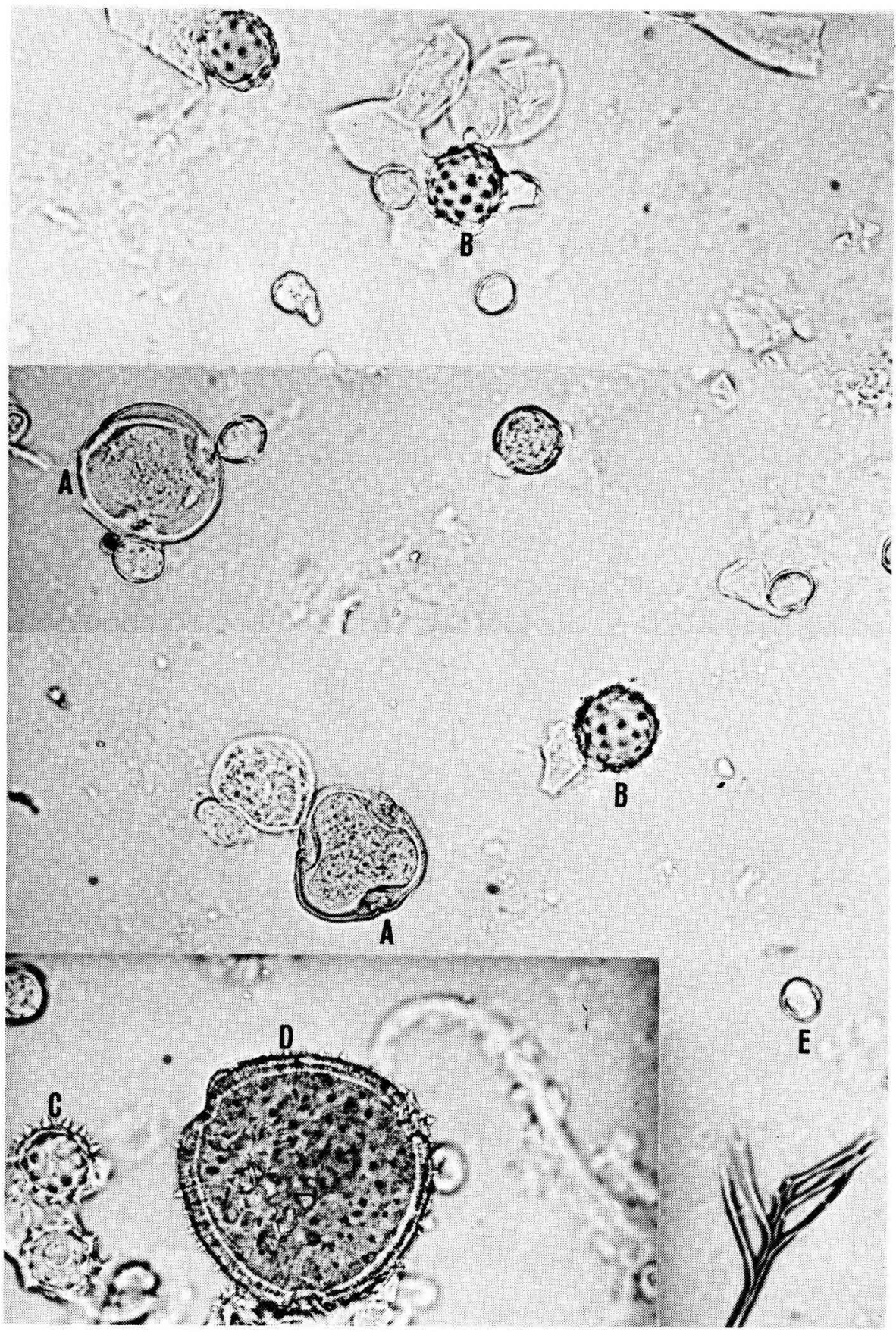

Fig. 10. - Spectre pollinique d'une gelée royale de Yougoslavie.

A) Tilia $s p . ; B$ ) Senecio $s p . ; C)$ Aster $s p . ; D)$ Dispacacée; E) Castanea sativa Mill. Авв. 10. - Pollenspektrum eines Weiselfuttersaftes aus Jugoslawien. 


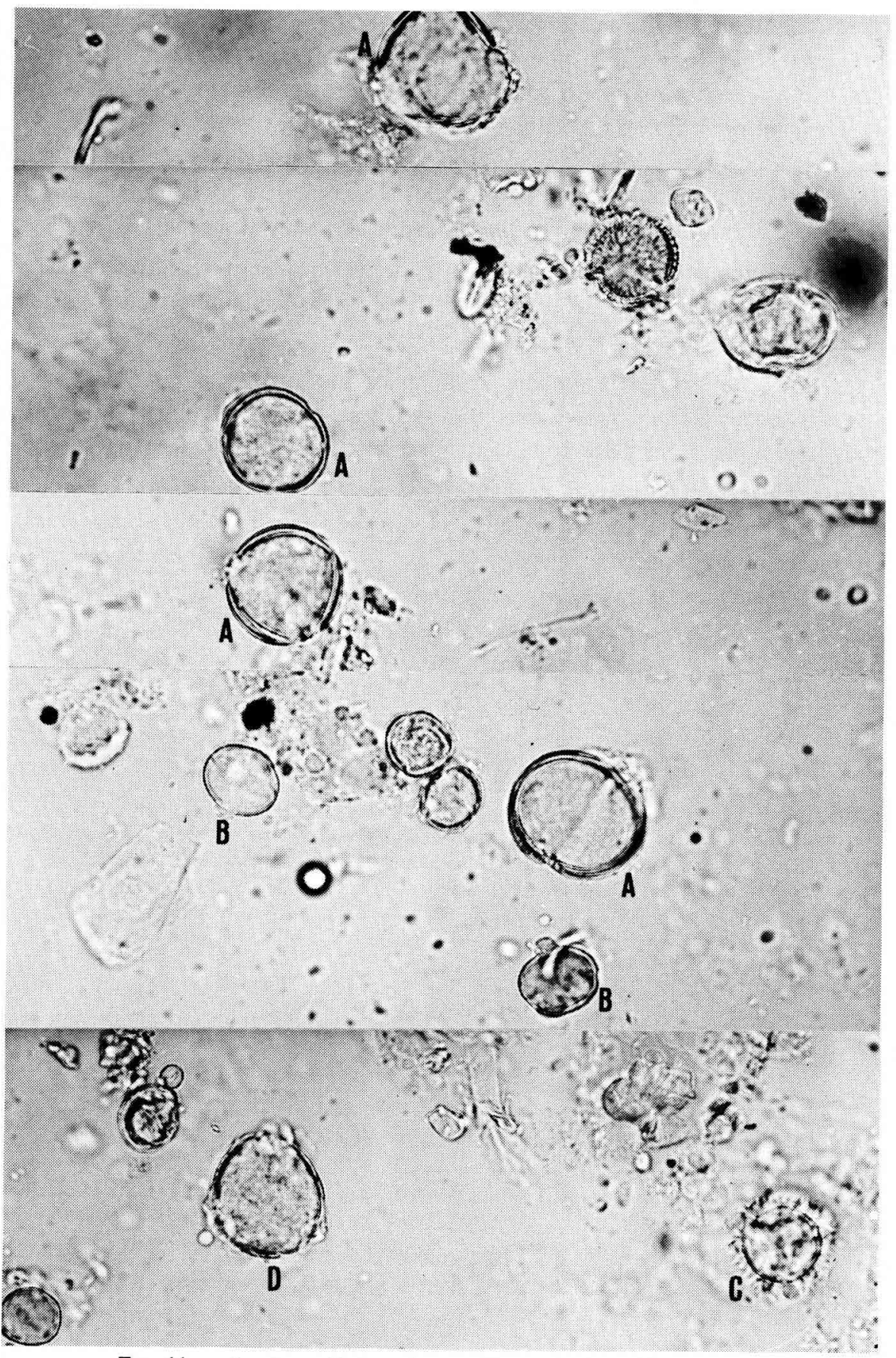

FIG. 11. - Spectre pollinique d'une gelée royale de Tchécoslovaquie.

A) Acer sp.; B) Trifolium repens $L . ; C)$ Taraxacum officinale Weber; D) Pirus $G r$.

Aв8. 11. - Pollenspektrum eines Weiselfuttersaftes aus der Tschechoslowakei. 
Les productions italiennes sont complètement différentes des autres et même celles qui proviennent de Russie, de Chine, du Mexique et du Texas, sont tout à fait typiques. On remarque quelques ressemblances dans les spectres polliniques des gelées royales qui proviennent des pays de l'Est Européen, à cause de la présence de quelques pollens communs tels que Centaurea Cyanus, Tilia, etc.; la différenciation est de toute façon possible si l'on considère les pourcentages élevés auxquels les pollens de Vicia, Acer, Helianthus, Compositae H., Polygonum, Fagopyrum sont présents.

Il faut quand même préciser que dans le cadre des productions analysées quelquesunes seulement (Italie, Chine, Texas, Bulgarie, France, Roumanie) sont obtenues en quantités commercialement valables.

Les résultats provenant de ces recherches confirment ce que LouvEaux et Chauvin avaient déjà mis en évidence en 1956, à savoir la possibilité d'utiliser l'analyse palynologique de la gelée royale pour remonter à son origine géographique.

On ne devrait pas avoir de problèmes même si le nombre des pays producteurs augmentait, étant donné que le principe qui inspire la méthode est pratiquement le même que celui appliqué aux miels.

On peut donc conclure que l'analyse pollinique peut être appliquée efficacement à la gelée royale, en particulier s'il s'avère nécessaire d'effectuer des contrôles de qualité pour sauvegarder le produit.

\section{REMERCIEMENTS}

On remercie vivement de leur aimable collaboration AMORIN (Texas), BorNus (Pologne), Burmstrov (Russie, Caucase), Cabrera Pech (Mexique), harnaj (Roumanie) Mihelic (Yougoslavie), Kopràcovà (Tchécoslovaquie); Piana (Italie), Simidtschev (Bulgarie), Smith (Canada).

\section{BIBLIOGRAPHIE}

Chauvin R., LouveauX J., 1956. - Étude macroscopique et microscopique de la gelée royale. L'Apiculteur, 33-35.

ELSER E., 1929. - Die chemische Zusammensetzung der Nahrungsstoffe der Biene. Märkische BienenZtg., 19, 210-215, 232-235, 248-252.

HA YDAK M. H., 1943. - Larval food and development of castes in the honey-bee. J. Econ. Entomol., 36, 778-792.

Planta A. von, 1888. - Über den Futtersaft der Bienen. Z. Physiol. Chem., 12, 327-354.

SMITH M. V., 1959. - Queen differentiation and the biologial testing of royal jelly. Cornell University agric. Expt. Sta. Memoir., 356, 56. 


\section{ZUSAMMENFASSUNG}

\section{DIE GEOGRAPHISCHE HERKUNFT DES WEISELFUTTERSAFTES}

Auf Grund einer von Louveaux und Chauvin 1956 durchgeführten Untersuchung war es uns möglich, 106 Weiselfuttersaft-Proben aus 12 verschiedenen Ländern (China, Mexico, Texas/U.S.A., Kanada, Russland, Polen, Rumänien, Jugoslawien, Tschechoslowakei, Bulgarien, Frankreich, Italien) pollenanalytisch zu, untersuchen.

Ziel der Untersuchung war es, den Nachweis zu erbringen, dass es möglich ist, die geographische Herkunft eines Weiselfuttersaftes anhand seines Pollenspektrums zu bestimmen.

Bei den Analysen wurde folgende Methode angewandt : $0,5 \mathrm{~g}$ Weiselfuttersaft wurden mit $8 \mathrm{ml}$ destilliertem Wasser homogenisiert, die Lösung durch Neutralisieren mit $5 \%$ iger $\mathrm{KOH}$ geklärt, danach zentrifugiert, dekantiert, mit einer $0,1 \quad \mathrm{n}-\mathrm{H}_{2} \mathrm{SO}_{4}$ Lösung gewaschen und nochmals zentrifugiert. Vom Sediment wurde das Präparat nach der offiziellen, von der Internationalen Kommission für Bienenbotanik für Honig festgelegten Methode angefertigt.

Wegen des mehr oder weniger hohen Prozentsatzes an deformierten oder zerbrochenen Pollenkörnern war die Pollenbestimmung nicht immer einfach. Durch die Untersuchung des Pollenspektrums, die sich hauptsächlich auf normale Pollenkörner bezog, wurde nachgewiesen, dass es möglich ist, Weiselfuttersäfte nach ihrem Herkunftsland zu unterscheiden.

In den meisten Fällen ist der Nachweis einfach. Bei manchen Ländern jedoch, z.B. osteuropäischen, treten ab und zu Schwierigkeiten auf. Daher erscheint eine gründlichere Untersuchung angebracht, ohne dass dadurch der Wert der Methode in Frage gestellt wäre. 